\title{
Analyse médico- légale dans les arts premiers et intégrité scientifique
}

\section{Sophie Jacqueline ${ }^{1}$, Philippe Charlier ${ }^{2}$}

> La communauté scientifique, dans son ensemble, se préoccupe de plus en plus des questions de déontologie et d'intégrité de la recherche. Au cours de notre travail de thèse portant sur les intérêts et les limites de l'étude des œuvres d'art premier, nous avons été amenés à réfléchir sur la place de l'intégrité scientifique dans nos recherches. Nous avons observé que celle-ci ainsi que ses manquements pouvaient impliquer différents acteurs : la communauté scientifique, mais également les communautés autochtones desquelles provenaient les œuvres que nous étudions, et la communauté du marché de l'art. Nous avons donc voulu comprendre comment un manquement à l'intégrité de la recherche pouvait être préjudiciable pour la science, mais également en affecter les différents acteurs. Pour cela, nous nous sommes intéressés à la circulation des œuvres d'art premier $^{1}$, et plus particulièrement à leur restitution, cette dernière faisant intervenir les différents acteurs que nous avons évoqués. Nous présentons ici une courte analyse de nos observations et quelques visées prospectives. <

\section{Le contexte des restitutions}

L'actualité de ces derniers mois au sujet de la restitution d'œuvres détenues par l'0ccident aux communautés autochtones, nous amène à rappeler les différentes catégories de demandes de restitutions qui existent et qui ne peuvent être confondues. Nous distinguerons ainsi les objets volés issus de campagnes de fouilles illégales, et les objets collectés aux alentours du XIXe siècle, dans le cadre de missions officielles.

De 1960 à 1970, les objets volés affluent sur les marchés occidentaux et, par voie de conséquences, dans les collections publiques et privées. C'est dans ce contexte que plusieurs źtats signent, en 1970, la convention de l'Organisation des Nations unies pour l'éducation, la science et la culture (UNESCO), qui déclare illicites l'importation, l'exportation et le transfert de propriété des biens culturels. Ces états s'engagent également à prendre les mesures appropriées, afin de saisir et de res-

${ }^{1}$ L'art « premier » ou art « primitif » est l'art des sociétés traditionnelles, sans écriture ou « primitives »

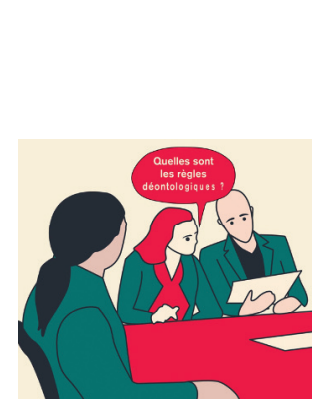

IUniversité VersaillesSaint-Quentin-en-yvelines, Laboratoire DANTE (EA4498), UFR des Sciences de la santé, 2 avenue de la source de la Bièvre,

78180 Montigny-Le-Bretonneux, France.

${ }^{2}$ Université VersaillesSaint- Quentin-en-yvelines, Laboratoire DANTE (EA4498),

tituer, à la requête de l'état d'origine, tout bien culturel volé et importé après la date d'entrée en vigueur de la convention, celle-ci n'ayant pas de caractère rétroactif $[1,2]$. Dès lors, plusieurs œuvres, répondant à la UFR des Sciences de la santé, 2 avenue de la source de la Bièvre, 78180 MontignyLe-Bretonneux, France, et Département de la Recherche et de l'Enseignement, Musée du quai Branly - Jacques Chirac, 222 rue de l'Université, 75007 Paris, France.

sophie.jacqueline14@gmail.com définition établie dans la convention, ont pu être restituées à leur pays d'origine. Dans certains cas, la disparition des œuvres était antérieure à 1970 ou les états responsables n'avaient pas ratifié le texte de la convention. L'esprit et les principes de ce traité ont néanmoins servi de base de réflexion, afin de permettre le retour des œuvres dans leur pays d'origine. Ainsi, en 2018, l'Allemagne a restitué plusieurs œuvres qu'elle détenait déjà avant 1970 : le couvercle d'un cercueil antique, rendu à l'Égypte, et neuf artefacts funéraires rendus à l'Alaska [3]. Il est important de noter que dans les deux cas, les pays sources ne demandaient la restitution que de quelques pièces à hautes valeurs culturelle, symbolique et artistique, ayant une signification particulière. Ils n'avaient pas exigé la restitution de l'ensemble des pièces conservées à l'étranger.

\section{Le cas des restes humains}

Parmi les objets d'art parvenus légalement dans les pays occidentaux, les restes humains constituent une catégo- 
rie particulière. Malgré le caractère « inaliénable », « insaisissable » et « imprescriptible » des œuvres conservées dans les musées français [4], il existe deux cas de restitution d'œuvres qui ont été rendues possibles par le vote d'une nouvelle loi, permettant le déclassement et le retour des pièces dans leur pays d'origine. Le premier concerne la restitution par la France à la Nouvelle Zélande, le 23 janvier 2012, de 21 têtes maories (mokomokai) collectées au XIX ${ }^{\mathrm{e}}$ siècle [5]; le second, la restitution à l'Afrique du Sud, en 2002, des restes de la « Vénus hottentote » ${ }^{1}$ [6].

\section{Réalisation et intégrité des expertises}

Au moment de la restitution d'œuvres, plusieurs questions peuvent se poser : à qui rendre ces œuvres ? Ou encore lesquelles faut-il restituer?

Dans le cas des têtes maories, une procédure médico-légale a été mise en place, en amont de la restitution, afin de distinguer le vrai du faux quant à ces têtes et ainsi ne pas manquer à l'intégrité scientifique [7]. Les visages maoris présentent de nombreux tatouages racontant l'histoire de l'individu et donc une partie de l'histoire de sa communauté. Les campagnes de restitutions peuvent s'appuyer sur ces tatouages afin de restituer les têtes à leurs propriétaires d'origine. Mais qu'en est-il lorsque certains tatouages ont été ajoutés post-mortem? Ne pas distinguer ces tatouages des originaux peut-il entraîner des identifications incorrectes des origines géographiques et tribales de l'individu qui les portait? C'est dans ce cas que les procédures médico-légales d'analyse de ces tatouages par des anthropologues, des archéologues, des médecins, des conservateurs, etc. prend tout son sens. II est ainsi particulièrement intéressant de déterminer le caractère ante-mortem ou post-mortem de chacun d'entre eux, afin que les mokomokai puissent être «rapatriées » correctement [7]. Mais plusieurs questions sont apparues au cours de ces analyses: par exemple, les têtes présentant des tatouages post-mortem ont-elles le même pouvoir et la même importance pour les communautés autochtones que les têtes présentant uniquement des tatouages ante-mortem? Conséquence de cette première question, est-il légitime de demander le rapatriement de ces têtes, qui pourraient être considérées comme « désacralisées ».

Un autre exemple de procédure médico-légale mise en place afin de statuer sur l'authenticité d'une œuvre concerne l'étude des têtes réduites jivaros ${ }^{2}$. Les collections occidentales actuelles ne renferment pas uniquement des têtes cérémonielles, mais également des têtes fabriquées par les Indiens autochtones à des fins commerciales. La restitution de ces têtes, à la demande des peuples du sud-est de l'Équateur et du nord du Pérou, pourrait donc poser problème. En effet, dans ce cas, quelles œuvres faudra-t-il restituer? Comment faire la différence entre tsantsa cérémonielles et tsantsa commerciales? L'examen médico-légal portant sur 20 têtes réduites a permis d'établir une première liste de 14 critères permettant d'établir leur authenticité (Tableau 1) [8]. De nombreuses études, fondées sur cet examen initial, ont ensuite étoffé cette liste de critères [9-11]. Ces analyses four-

${ }^{1}$ Saartjie Baartman de son vrai nom Sawtche, surnommée la Vénus Hottentote, serait née aux alentours de 1789 dans l'actuelle Afrique du Sud. Elle sera emmenée d'abord à Londres en 1810 pour être exhibée, puis à Paris, oû elle meurt le 29 décembre 1815.

${ }^{2}$ Les têtes réduites ou tsantsa sont réalisées à partir de têtes humaines par des tribus Jivaros d'Amérique du Sud. nissent désormais des données solides, qui permettent le rapatriement de ces restes humains authentifiés aux communautés autochtones.

\section{L'interconnexion de trois systèmes}

Nous avons évoqué quelques exemples du travail de la communauté scientifique et les questions auxquelles celle-ci devait répondre. Mais, qu'en est-il des communautés autochtones? Elles sont bien sûr concernées par ces questions de restitution, sans oublier qu'elles sont également concernées par le travail des scientifiques, ce qui nous conduit à nous interroger sur l'éthique de l'utilisation des outils médico-légaux. Les examens par imagerie médicale sont non invasifs et non destructeurs, il n'y a donc pas d'altération physique de l'objet examiné. L'intégrité physique de l'œuvre est donc préservée. Mais en est-il de même pour ce qui est de l'intégrité morale associée à ces pièces? Certaines réalisations, telles que les boli du Mali ${ }^{3}$, sont éminemment sacrées et conservées à l'abri des regards. Or, les examens effectués sur ces objets permettent de plonger à l'intérieur de l'œuvre, de la «mettre à nu » et d'approcher une partie des secrets de sa composition, sans toutefois révéler l'intégralité des gestes ayant conduit à son élaboration. Nous pouvons alors nous demander si notre regard n'est pas indiscret, voire indécent, et si nous n'avons pas, en quelque sorte, violé le secret de ces objets. L'objet ne perd-il pas, lors de son étude, son caractère sacré, pour être réduit à un usage profane. Ne participons-nous pas à l'exécration de l'objet en le désacralisant?

La question du lien entre l'œuvre et son créateur nécessite donc d'être abordée. Le créateur a en effet le droit de s'opposer aux atteintes portées à l'intégrité de son $œ u v r e$ lors de l'analyse. L'assentiment et la participation des représentants de chaque culture aux études menées doivent donc être pris en considération, afin de décider ce qu'il est possible ou non d'entreprendre. Nous devons donc nous demander jusqu'où il est possible d'aller dans l'analyse. Si, en appliquant les progrès des connaissances et en utilisant abusivement les outils biomédicaux, on viole l'intégrité physique ou morale d'un objet, nous pouvons nous demander si ce nonrespect de l'œuvre ou de son propriétaire ne relève pas d'un manquement à l'intégrité scientifique.

Enfin, n'oublions pas la communauté des marchands. En effet, les œuvres étudiées et susceptibles d'être restituées sont, pour la plupart, passées ou passeront entre les mains de cette communauté des marchands

${ }^{3} \mathrm{Au} \mathrm{Mali}$, un boli est un objet source de pouvoirs extraordinaires et auquel on rend un culte. 


\begin{tabular}{|c|c|}
\hline 1 & $\begin{array}{l}\text { Couleur de peau sombre, noire, ou marron, due à l'imprégnation par } \\
\text { la poussière de charbon de bois lors de la fabrication }\end{array}$ \\
\hline 2 & $\begin{array}{l}\text { Fibres végétales maintenant les lèvres (ou si absentes ou enlevées } \\
\text { lors de la production, présence des trous correspondants alignés } \\
\text { verticalement derrière les lèvres supérieures et inférieures) }\end{array}$ \\
\hline 3 & $\begin{array}{l}\text { Boucle de bois ou de fibres cousue au niveau du cou (si absente ou } \\
\text { retirée, des traces de sutures ou de sciage persistent) }\end{array}$ \\
\hline 4 & Épaisseur importante au niveau de la nuque \\
\hline 5 & $\begin{array}{l}\text { Forme ovale du cou en section transversale ou compression latérale } \\
\text { de la tête }\end{array}$ \\
\hline 6 & $\begin{array}{l}\text { A l'arrière, les tissus sont maintenus ensemble par une suture de } \\
\text { fibres }\end{array}$ \\
\hline 7 & $\begin{array}{l}\text { Conservation des détails anatomiques de l'oreille avec possibilité de } \\
\text { trous dans l'oreille, remplis ou non par une cheville de bois }\end{array}$ \\
\hline 8 & Yeux fermés (avec possibilité de couture de l'intérieur) \\
\hline 9 & Présence de poils dans les narines \\
\hline 10 & Longs cheveux noirs (ou coupés après le processus de rétrécissement) \\
\hline 11 & $\begin{array}{l}\text { Longue suspension en corde au sommet de la tête (ou si absente, } \\
\text { présence de trous en relation) }\end{array}$ \\
\hline 12 & Absence de peinture faciale ou de tatouage ethnique \\
\hline 13 & Absence de fragments crâniens persistants \\
\hline 14 & Remplissage des cavités internes par du sable ou des charbons \\
\hline
\end{tabular}

Tableau I. Liste des 14 critères diagnostiques pour l'authentification des tsantsa.

d'art, que ce soit en salles des ventes ou dans des galeries, et la valeur de l'objet dépendra de son authentification. Le travail de la communauté scientifique est donc particulièrement important. Si nous reprenons le cas des tsantsa, leur valeur ne sera pas la même, selon qu'elle a un caractère cérémoniel ou commercial. Un lot de trois têtes réduites, de style jivaros, en peau de chèvre a été estimé entre 100 et 150 euros et vendu 128 euros en octobre 2017 à Lyon [12]. Une tête réduite authentifiée a été, elle, estimée entre 6000 et 10000 euros en novembre 2009 à Paris, et a été adjugée 14870 euros [13]. Une question se pose alors : qui la Science doit-elle servir : la vérité ou des intérêts financiers qui ne la concernent pas?

Une interconnexion entre les trois systèmes que sont les marchands d'art, les scientifiques et les peuples autochtones apparaît donc, et un manquement à l'intégrité de la recherche peut avoir des répercussions sur chacun des trois.

\section{Conclusion}

Une des conséquences directes du rapatriement est une augmentation significative du nombre de demandes d'authentification. Nous remarquons que l'intégrité concerne plusieurs facettes de ces exper- tises, à savoir la conduite, la réalisation et la diffusion des résultats de l'étude. À ces différents niveaux, les manquements à l'intégrité peuvent être nombreux et engendrer des préjudices aux différentes communautés impliquées. Ainsi les conséquences peuvent être aussi bien morales que financières. L'intégrité scientifique est indissociable de l'excellence technique, de la justesse de la restitution à l'ethnie la plus concernée par l'artefact, au service du devoir de mémoire, mais aussi de l'équité sur le plan patrimonial et géopolitique. Elle se trouve ici à la croisée des chemins entre le droit international, les sciences humaines, et les sciences fondamentales. L'intégrité scientifique de la recherche doit donc pour nous être une préoccupation constante pendant les différentes étapes de nos travaux. $\diamond$ Forensic analysis in tribal art and scientific integrity

\section{LIENS D’INTÉR̂̂T}

Les auteurs déclarent n'avoir aucun lien d'intérêt concernant les données publiées dans cet article.

\section{RÉEÉRENCES}

1. Organisation des Nations Unies pour l'éducation, la science et la culture. Texte de la Convention de 1970. http://www.unesco.org/new/fr/culture/ themes/illicit-trafficking-of-cultural-property/1970-convention/textof-the-convention/

2. Organisation des Nations Unies pour l'éducation, la science et la culture. Autres cas de retour ou de restitution de biens culturels. http://www. unesco.org/new/fr/culture/themes/illicit-trafficking-of-culturalproperty/other-cases-of-return-or-restitution-of-cultural-objects/

3. Organisation des Nations Unies pour l'éducation, la science et la culture. Cas récents de restitution en application de la Convention de 1970. http://www.unesco.org/new/fr/culture/themes/illicit-trafficking-ofcultural-property/recent-restitution-cases-of-cultural-objects-usingthe-1970-convention/

4. Loi $n^{\circ} 2002-5$ du 4 janvier 2002 relative aux musées de France 2002.

5. Loi n 2010-501 du 18 mai 2010 visant à autoriser la restitution par la France des têtes maories à la Nouvelle-Zélande et relative à la gestion des collections 2010 .

6. Loi $n^{\circ} 2002-323$ du 6 mars 2002 relative à la restitution par la France de la dépouille mortelle de Saartjie Baartman à l'Afrique du Sud 2002.

7. Charlier P, Huynh-Charlier I, Brun L, et al. Maori heads (mokomokai): the usefulness of a complete forensic analysis procedure. Forensic Sci Med Pathol $2014 ; 10$ : 371-9.

8. Charlier P, Huynh-Charlier I, Brun L, et al. Shrunken head (tsantsa): a complete forensic analysis procedure. Forensic Sci Int $2012 ; 222: 399$ el-5.

9. Houlton TMR. A morphometric investigation into shrunken heads. J Cultural Heritage $2018 ; 32: 238-47$.

10. Houlton TMR, Wilkinson C. Facial preservation following extreme mummification: Shrunken heads. Forensic Sci Int 2018; 286 : 31-41.

11. Houlton TMR, Wilkinson CM. Recently identified features that help to distinguish ceremonial tsantsa from commercial shrunken heads. J Cultural Heritage $2016 ; 20: 660-70$.

12. Venezuela : un ensemble de trois têtes réduites ou Tsantsa en peau de chèvre. https://www.aguttes.com

13. Pérou : tête réduite jivaro. https://www.artkhade.com/
TIRÉS À PART

S. Jacqueline 